\title{
CHEWING XYLITOL GUM COULD ACCELERATE BOWEL MOTILITY RECOVERY AFTER ELECTIVE OPEN PROCTECTOMY FOR RECTAL CANCER
}

\author{
Ping Yang*, Wu Jun Long and Wei LI
}

Department of Gastrointestinal Surgery, The East Branch of Sichuan Academy of Medical Sciences \& Sichuan Provingial People's Hospital, University of Electronic Science and Technology of China Affiliated Hospital \& Sichuan Provincial $\frac{\ulcorner}{}$ People's Hospital, Chengdu 610110, Sichuan Province, China

\begin{abstract}
Background: A number of studies with conflicting results have evaluated the effect of chewing gum on post-operative gastrointestinal recovery in patients after major colorectal surgery. Objective: The objective of the study was to study the efficacy of chewing gum in patients with rectal cancer after elective open proctectomy only. Methods: A randomized controlled clinical trial was performed. We recruited patients who would undergo elective open proctectomy for rectal cancer in Sichuan Academy of Medical Sciences and Sichuan Provincial People's Hospital. Patients in the intervention arm received chewing gum 3 times a day postoperatively. All patients in the trial were placed on the same perioperative management and standardized post-operative care plans. The primary outcome was time to the first peristalsis sounds, time to first flatus and the first defecation. Results $\_A$ total of 89 patients were recruited. The time to the first flatus was $42.33 \pm 3.46 \mathrm{~h}$ in the gum group and $49.20 \pm 1.42 \mathrm{~h}$ in the control group ( $p<0.05$ ). The time to the first defecation was significantly shorter in the gum-chewing group than in the controf group $(66.07 \pm 2.36$ vs. $78.37 \pm 1.62 \mathrm{~h} ; \mathrm{p}<0.05)$. Post-operative ileus (POI) was confirmed in 2 patients in the gum-chewing group and in 7 in the control group ( $7.0 \%$ vs. $23.9 \%$; $=0.028$ ). Discussion: The present study suggests that chewing gum is a method to reduce the time to first flatus, time to first defecation and $\mathrm{POI}$ in patients undergoing elective open proctectomy for rectaPcancer.
\end{abstract}

Key words: Chewing gum. Rectal cancer. Rectal surgery.

\section{INTRODUCTION}

Post-operative ileus (POI) is a well-known complication, with an incidence of $14 \%$ in colorectal surgery ${ }^{1}$. $\mathrm{POI}$ is defined as "two or more episodes of nausea/ vomiting, inability to tolerate oral diet over $24 \mathrm{~h}$, absence of flatus over $24 \mathrm{~h}$, distention and radiologic

\section{Corresponding author:}

*Ping Yang

Department of Gastrointestinal Surgery

The East Branch of Sichuan Academy of Medical Sciences \& Sichuan Provincial People's Hospital

University of Electronic Science and Technology of China Affiliated Hospital \& Sichuan Provincial People's Hospital 585 Honghe North Road

Chengdu 610110, Sichuan Province, China

E-mail:Yp29548928@163.com confirmation on or after post-operative day 4 without prior resolution ${ }^{2}$." The risk factors for the development of POI were identified as follows: smoking, weight loss, pre-operative oral antibiotics, mechanical bowel preparation, and surgical approach ${ }^{1}$. Common ${ }^{\circ} y m p-$ toms of $\mathrm{POI}$ are vomiting, nausea, and abdominal pain. However, prolonged POI can decrease patient cổmfort

Received for publication: 07-10-2017

Approved for publication: 10-01-2018 doi: $10.24875 / R I C .18002428$ 
and increase morbidity and mortality, prolong hospital stay, and raise healthcare costs ${ }^{3,4}$. Implementation of fast-track colorectal surgical programs has already shown promising results in reducing overall hospital stay ${ }^{5,6}$. Further reduction of this post-operative transient episode of gastrointestinal hypomotility will contribute to the enhanced recovery after colorectal surgery.

In clinical practice, there is a lack of effective strategies for the prevention and treatment of POI. Several strategies and interventions have been tested to prevent or reduce $\mathrm{POI}$, both pharmacological and non-pharmacological. One of these strategies is gum chewing, a new and simple modality, which can accelerate complication-free recovery of gastrointestinal function following colorectal surgery ${ }^{7-9}$, although the underlying mechanism remains unclear. Even though several studies have shown beneficial effects on recovery after different types of surgery, no study has yet investigated the effect of chewing gum treatment on recovery after elective open proctectomy for rectal cancer surgery only.

To examine this question, we conducted a randomized controlled trial (RCT) to investigate the effects of chewing xylitol gum treatment on patients undergoing elective open proctectomy for rectal cancer.

\section{METHODS}

\section{Patients}

The present study was conducted in October 2013. Patients older than 18 years who underwent elective open proctectomy for rectal cancer at East Ward Gastrointestinal Surgery Departments of the Sichuan Academy of Medical Sciences and Sichuan Provincial People's Hospital were eligible to participate in the study. Diagnosis of rectal cancer was based on histopathological examination of endoscopic biopsies. Patients were excluded if they had a history of abdominal surgery, inflammatory bowel disease, abdominal radiation, or substance abuse; if they had psychological or social conditions that might interfere with their participation in the study; if they were allergic to xylitol gum; or if they required intensive care more than $24 \mathrm{~h}$ postoperatively or a nasogastric tube beyond the first post-operative morning.

\section{Study design}

This trial is designed as a prospective RCT. The study information was explained to all enrolled patients. This study was approved by the Ethics Committee of the Sichuan Academy of Medical Sciences and Sichuan Provincial People's Hospital (YW2013-016) After obtaining written informed consent patients were randomly assigned to one of two groups by the TenAlea software (http://nl.tenalea.net). Randomization was performed the day before proctectomy. The commanding researcher assigned the participants to the test group or control group using a sealed ôpaque envelope to conceal the random allocation seguence until interventions were assigned. The treatment group received chewing xylitol gum and standa care. Controls received standard care only. The fundamentally different characteristics of two groups meant that no blinding was used after randomization. Patients were informed that the effect of chewing xylitol gum to promote recovery of gastrointestinal function after proctectomy was not known and that this measure was not expected to cause obvious side effects.

\section{Procedure}

The same surgical team performed all proctectomies using general anesthesia, and the same evidence based protocol of perioperative management and ständardized post-operative care plans were used. All patients enrolled in both groups underwent low anterior resection of rectal cancer. A pelvic cavity drainage tube was placed in each patient. The nasogastric decomp ression tube was removed until after passage of first flatus. Enteral or nasogastric feeding was not provided until after passage of first flatus. All patients received a proton pump inhibitor for 3 days after proctectomy surgery. All patients were mobilized as soon a's-possible in the post-operative period. We used visual analog scale to evaluate post-operative pain in patients. If the score was $>8$, patients received an intramuscular injection of pethidine hydrochloride, each dose of 50-100 mg. Patient characteristics and clinical parameters were registered in an electronic database by the specialized nursing ward staff. Primary endpoinss were time to first flatus and time to first defecation, which were recorded daily by nursing staff. 
This study used a commercially available Orange Mint flavored xylitol gum (Wrigley Sugar Co., Ltd, Shanghai, China). The gum ( $2.8 \mathrm{~g})$ contained $2.4 \mathrm{~g}$ carbohydrates, $1 \mathrm{mg}$ sodium, and xylitol accounts for $36 \%$ of the added amount. The test group started to chew xylitol gum on the $1^{\text {st }}$ day after proctectomy surgery, two pieces of gum for 30 min 3 times a day, during three intervals $(08.00-12.00,12.00-16.00$, and 16.00-20.00 h).

\section{Sample size calculation}

The sample size was calculated by statistical software, based on the mean time to first flatus which was assumed to be 73 and $51 \mathrm{~h}$ in both groups ${ }^{10}$. The minimum detectable difference was 22. Assuming that the common standard deviation is $24 \mathrm{~h}$, the sample size was calculated to be a total of 100 participants, applying a statistical power of $90 \%$ at a two-sided significance level of $5 \%$. An additional, 10 subjects were recruited to offset potential attrition.

\section{Statistical analysis}

SPSS 22.0 was used for all statistical analyses, with the statistical significance defined as a two-tailed $p<0.05$. Data for continuous variables were expressed as median (range), while data for categorical variables were expressed as number (percentage). Demographic differences between the control and test groups were ascertained using the chi-squared test for categorical variables and the Mann-Whitney U-test for continuous variables.

\section{RESULTS}

\section{Patient characteristics}

From October 1, 2013, to July 31, 2017, 153 patients with rectal cancer were assessed for eligibility. Of these, 43 were excluded because they did not meet the inclusion criteria, 12 refused to participate and 5 were unwilling to receive chewing gum. The remaining 110 patients were randomly assigned tôtreatment group or control group. After randomizätion, 5 patients were excluded because they had a profonged stay on the intensive care unit, and another 3 patients from the treatment group were excluded beccause they were incapable of chewing gum. Seven patients discontinued the study, and 6 received the incorrect intervention during the study period, so these 13 were excluded from the final analysis. In the end, 89 patients completed the study, comprising 43 in the treatment group and 46 in the control group (Fig. 1).

Patients in both groups had similar clinical and proctectomy features, including gender, age, body mass index, duration of surgery, and blood loss (Table 1). There were no intervention-related adverse eyents more severe than Grade I recorded in any of the groups, based on the Common Terminology Ériteria for Adverse Events 3.011.

\section{Post-operative bowel function recovery results}

The time to first flatus was $42.33 \pm 3.46$ hoin the gum-chewing group and $49.20 \pm 1.42 \mathrm{~h}$ in the $\frac{\bar{\sigma}}{6}$ ontrol

Table 1. Clinicopathological data of patients with rectal cancer in chewing gum group and control group

\begin{tabular}{|c|c|c|c|c|}
\hline & & & & \\
\hline Variable & Chewing gum group (mean \pm SD) & Control group (mean \pm SD) & 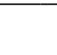 & $\mathrm{p}$ \\
\hline Age (years) & $62.70 \pm 7.84$ & $63.37 \pm 7.23$ & $\frac{\varsigma}{6} 0$ & .675 \\
\hline Male/Female & $31 / 12$ & $30 / 16$ & \pm 0 & .485 \\
\hline $\mathrm{BMI}$ & $21.88 \pm 2.37$ & $21.92 \pm 2.00$ & $\cup 0$ & .936 \\
\hline Smoking & 21 & 25 & 응 0 & .603 \\
\hline Alcohol use & 7 & 7 & 50 & .891 \\
\hline Surgical time (min) & $122.42 \pm 14.38$ & $126.39 \pm 15.11$ & $\sim 0$ & .208 \\
\hline Blood loss (mL) & $151.40 \pm 16.12$ & $145.43 \pm 14.86$ & 三0 & .073 \\
\hline TNM staging & & & $\stackrel{+}{4}$ & \\
\hline I & 12 & 13 & 00 & .999 \\
\hline ॥ & 10 & 11 & $t$ & \\
\hline III & 12 & 13 & 으 & \\
\hline IV & 9 & 9 & 0 & \\
\hline BMI: body mass inde & nor-node-metastasis, SD: standard & & & \\
\hline
\end{tabular}


Figure 1. Consort diagram for the study.

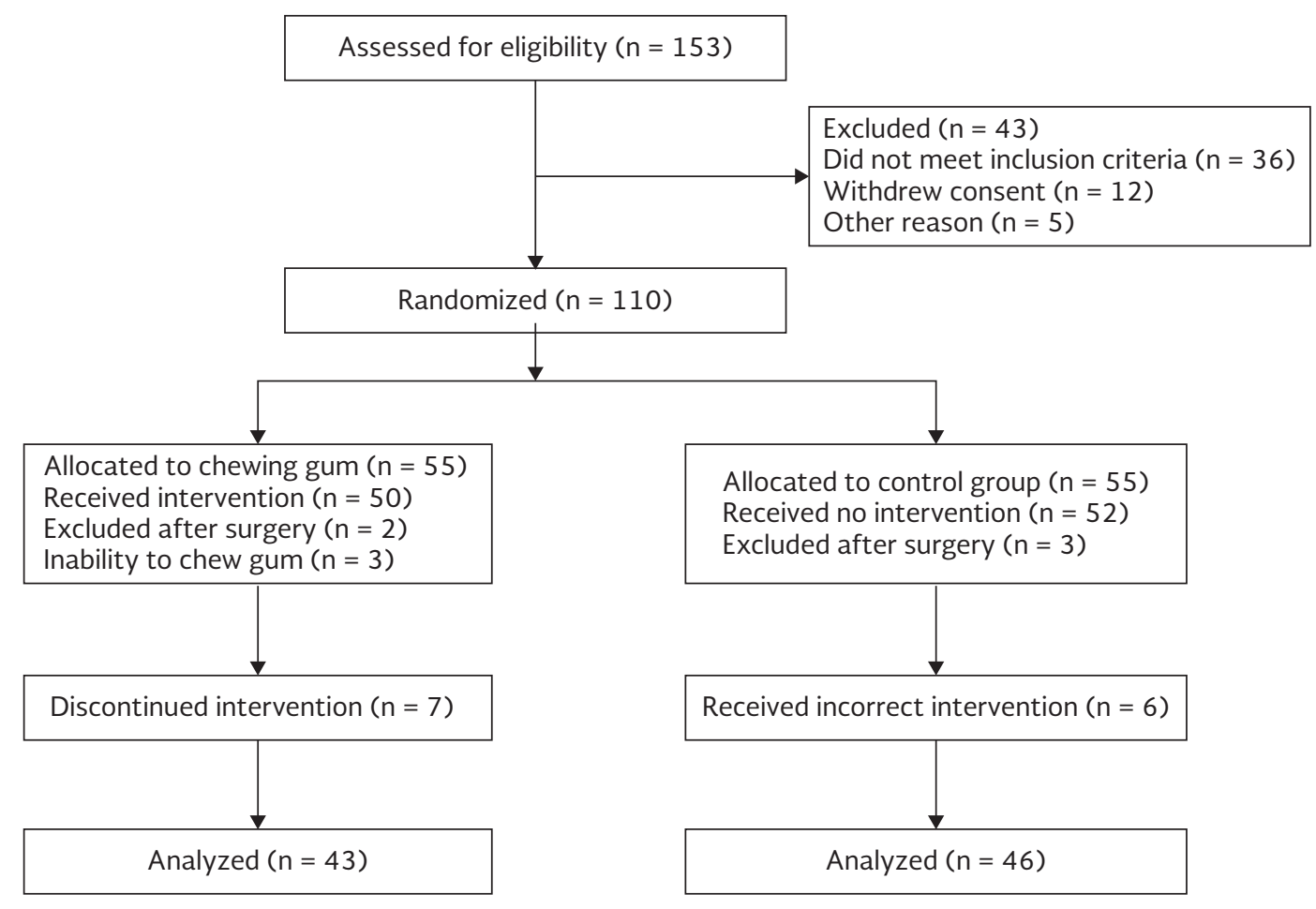

group ( $p=0.0001$ ). The time to first defecation was significantly shorter in the gum-chewing group than in controls $(p=0.0001)$ (Table 2$)$. There were no adverse events more severe than Grade I.

\section{Post-operative opioid analgesic use}

The use of opioid analgesic was $160.47 \pm 9.49 \mathrm{mg}$ in the gum-chewing group and $200.00 \pm 9.83 \mathrm{mg}$ in the control group $(p=0.005)$ (Table 2$)$.

\section{Post-operative complications}

$\mathrm{POI}$ was confirmed in 2 patients of the gum-chewing group and in 7 controls ( $7.0 \%$ vs. $23.9 \% ; p=0.028$ ). Abdominal bloating was reported in 10 subjects of the gum-chewing group and 13 controls, respectively $(23.3 \%$ vs. $28.3 \% ; p=0.590)$. Nausea was reported in 6 patients from the gum-chewing group and 11 from the control group ( $13.9 \%$ vs. $23.9 \%$; $p=0.232$ ); vomiting was present in 5 patients from the gum-chewing group and in 9 controls ( $11.6 \%$ vs. $19.6 \%$; $p=$ $0.304)$. There were two cases of anastomotic leakage in each group ( $4.7 \%$ vs. $4.3 \% ; p=0.945$ ). Pneumonia

was observed in 2 patients from the gum-ciewing group and 3 from the control group $(4.7 \%$ vs? $6.5 \%$; $\mathrm{p}=0.702$ ). Incision infection's rate was $4.7 \%$ and $6.5 \%$ in the gum-chewing group and the controP group $(p=0.702)$ (Table 3$)$.

\section{DISCUSSION}

Interventions with the potential to limit the discomfort of $\mathrm{POI}$ are welcomed, especially with the increasing pressure due to the limits in healthcare costs and the continuous need to improve the quality of the pâtients' perioperative period experience. Various strattegies have been developed to reduce the incidence of this complication ${ }^{12,13}$, including fast track care, minimally invasive surgery, epidural anesthesia, probiotics, and early feeding in combination with multimodal regimens, but none of these methods is entirely satisfactory. A new and simple modality is gum chewing, which can accelerate complication-free recovery of gastrointestinal function following colorectal surgery. The previous research ${ }^{7,8}$ had focused primarily on colon surgery, with beneficial results on the duration of POI. Therejis also a study showing that chewing gum does not hasten the return of gastrointestinal function in patients who 
Table 2. Outcomes of post-operative chewing gum group and control group in patients with rectal cancer

\begin{tabular}{|c|c|c|c|}
\hline Variable & Chewing gum group $(n=43)$ & Control group $(n=46)$ & $\varepsilon$ \\
\hline Time to first flatus (h) & $42.33 \pm 3.46$ & $49.20 \pm 1.42$ & 0.0001 \\
\hline Time to first defecation $(\mathrm{h})$ & $66.07 \pm 2.36$ & $78.37 \pm 1.62$ & (c).0001 \\
\hline Post-operative opioid analgesic use (mg) & $160.47 \pm 9.49$ & $200.00 \pm 9.83$ & 0.005 \\
\hline
\end{tabular}

Table 3. Post-operative complications in chewing gum group and control group

\begin{tabular}{|c|c|c|c|c|}
\hline Variable & Chewing gum group $(n=43)$ & Control group $(n=46)$ & 을 & $\mathrm{p}$ \\
\hline Post-operative ileus & $3(7.0 \%)$ & $11(23.9 \%)$ & & 0.028 \\
\hline Abdominal bloating & $10(23.3 \%)$ & $13(28.3 \%)$ & 40 & .590 \\
\hline Nausea & $6(13.9 \%)$ & $11(23.9 \%)$ & & .232 \\
\hline Vomiting & $5(11.6 \%)$ & $9(19.6 \%)$ & & .304 \\
\hline Pneumonia & $2(4.7 \%)$ & $3(6.5 \%)$ & & .702 \\
\hline Incision infection & $2(4.7 \%)$ & $3(6.5 \%)$ & $\equiv 0$ & .702 \\
\hline Abdominal abscess & $1(2.3 \%)$ & $2(4.3 \%)$ & & .579 \\
\hline Anastomotic leakage & $2(4.7 \%)$ & $2(4.3 \%)$ & ○0 & 0.945 \\
\hline
\end{tabular}

receive accelerated post-operative feeding ${ }^{14}$. However, our results suggest that chewing gum could shorten the recovery time of a post-operative gastrointestinal function in elective open proctectomy for rectal cancer.

In our randomized controlled study, the time to first flatus and defecation was shorter in the control group. This suggests that the chewing gum did hasten the return of gastrointestinal function, which is supported by findings in other randomized trials ${ }^{8,15}$ and meta-analyses ${ }^{16}$. In the study by Vergara-Fernandez et al. ${ }^{15}$, the passage of flatus within the first $48 \mathrm{~h}$ was present in 30 patients from Group A and in 20 from Group B ( $94 \%$ vs. $63 \%$ ). Similar results were obtained in a meta-analysis ${ }^{16}$ which included 13 RCTs enrolling 993 patients, with 499 patients in the chewing gum group and 494 patients in the control group. These results revealed that chewing gum could significantly reduce the time to first passage of flatus and the time to the first defecation after colorectal surgery.

Vergara-Fernandez et al. ${ }^{15}$ reported POI in 6\% (2/32) in the gum-chewing group and in $21.8 \%(7 / 32)$ in the standard post-operative recovery group. In our study, $\mathrm{POI}$ was confirmed in 2 patients of the gum-chewing group and in 7 of the control group. In terms of post-operative anastomotic leakage, pneumonia, and incision infection, there were no statistical differences between the two groups. Our findings are similar to the results of this study ${ }^{15}$.

Gastrointestinal function requires a certain time from the post-operative inhibition to return to normal function. If the patients without flatus for a protonged time after surgery there can be bloating, intestinal paralysis, intestinal adhesions, and other complications, affect the post-operative diet and bowel functional recovery ${ }^{17}$. Due to the surgical trauma, anesthesia suppression, and other added factors, pattients undergoing gastrointestinal surgery have different degrees of gastrointestinal dysfunction, ${ }^{18-20}$ of which intestinal inflammation induced by operative trauma appears to be the most frequent. ${ }^{21}$ Inflammato y infiltration into the intestinal muscularis can lead to hypomotility along the entire gastrointestinal tract ${ }^{22,23}$. Consistent with this, early inhibition of inflammation reduces the incidence of $\mathrm{POI}$, usually by vagus nerve-mediated activation of the autonomic nervous system ${ }^{22,24}$. Based on the sham feeding theory, post-operative gum chewing can effectively stimulate the mouth by imitating the diet, promote neurohumoral reflex, increase gastrointestinal fluid secretion, promote gastrointestinal motility, and improve gastrointestinal function recovery ${ }^{8,25}$. This may help explain why we observed that gum chewing promoted frecovery of gastrointestinal function, although the exact mechanism of chewing gum is not clear until now.

The results from our study should be intep reted with caution. There are some limitations in this study, although the frequencies of these treatments were similar between the two groups. One is the pack of blinding, which was not feasible because of the nature of the interventions. Another limitation was that patients within each group differed in whether they received opioid analgesia, which may have confounded 
our analysis. In our study, the gum-chewing group was given fewer opioid analgesics postoperatively than the control group, and the exact reason for this discrepancy was unclear, probably because chewing gum distracted the patient's attention from pain. Because radiation therapy can cause colonic or rectal wall thickening and colonic strictures ${ }^{26}$, this can lead to intestinal obstruction. Considering these complications, we excluded patients with pre-operative radiotherapy. However, this may have led to selection bias in this study.

In conclusion, the present study suggests that chewing gum is a method for decreasing the time to first flatus, time to first defecation and $\mathrm{POI}$ in patients undergoing elective open proctectomy for rectal cancer. Chewing gum can be added to post-proctectomy for rectal cancer care without any concern for early post-operation feeding as a low-cost, safe, and tolerable treatment in early intestinal stimulation. Future studies should explore whether chewing gum can treat POI after it has already developed.

\section{REFERENCES}

1. Murphy MM, Tevis SE, Kennedy GD. Independent risk factors for prolonged postoperative ileus development. J Surg Res. 2016;201:279-85

2. Vather R, Trivedi S, Bissett I. Defining postoperative ileus: Results of a systematic review and global survey. J Gastrointest Surg. 2013;17:962-72.

3. Earnshaw SR, Kauf TL, McDade C, et al. Economic impact of alvimopan considering varying definitions of postoperative ileus. ] Am Coll Surg. 2015;221:941-50.

4. Asgeirsson T, El-Badawi KI, Mahmood A, et al. Postoperative ileus: It costs more than you expect. J Am Coll Surg. 2010;210: 228-31.

5. Compagna R, Aprea G, De Rosa D, et al. Fast track for elderly patients: Is it feasible for colorectal surgery? Int J Surg. 2014;12 Suppl 2:S20-2.

6. Zhao JH, Sun JX, Gao P, et al. Fast-track surgery versus traditional perioperative care in laparoscopic colorectal cancer surgery: A meta-analysis. BMC Cancer. 2014;14:607.

7. Kobayashi T, Masaki T, Kogawa K, Matsuoka H, Sugiyama M. Efficacy of gum chewing on bowel movement after open colectomy for left-sided colorectal cancer: A randomized clinical trial. Dis Colon Rectum. 2015;58:1058-63.

8. van den Heijkant TC, Costes LM, van der Lee DG, et al. Randomized clinical trial of the effe ct of gum chewing on postoperative ileus and inflammation in colorectal surgery. $\mathrm{Br}$ J Surg. 2015;102:202-11.
9. Topcu SY, Oztekin SD. Effect of gum chewing on reducing postoperative ileus and recovery after colorectal surgery. $\mathrm{A}$ randomised controlled trial. Complement Ther Cliñ Pract. 2016;23:21-5

10. Pang $\mathrm{YH}, \mathrm{Ma} \mathrm{L}, \mathrm{Mao} \mathrm{CL}$, et al. Influence of acupoint therapy with simo decoction on postoperative gastrointestinal Gunction recovery of primary liver cancer patients. J Nurs (China). 2013;20:53-5.

11. Trotti A, Colevas AD, Setser A, et al. CTCAE v3.0: Development of a comprehensive grading system for the adverse effects of cancer treatment. Semin Radiat Oncol. 2003;13:176-81.

12. Zhang Z, Wang C, Li Q, et al. Electroacupuncture at ST 36 accelerates the recovery of gastrointestinal motility after colorectal surgery: A randomised controlled trial. Acupunct Med. $2014 ; 32$ 223-6.

13. Wallström A, Frisman GH. Facilitating early recovery @f bowel motility after colorectal surgery: A systematic review. J Clin Nurs. 2014;23:24-44

14. Lim P, Morris OJ, Nolan G, et al. Sham feeding with chewing gum after elective colorectal resectional surgery: A randomized clinical trial. Ann Surg. 2013;257:1016-24.

15. Vergara-Fernandez O, Gonzalez-Vargas AP, CastellanosJuarez JC, Salgado-Nesme N, Sanchez-Garcia Ramos E. Usefulness of gum chewing to decrease postoperative ileus in colorectal surgery with primary anastomosis: A Randomized controlled trial. Rev Invest Clin. 2016;68:314-8.

16. Wang XJ, Chi P. Effect of chewing gum on the promotion of intestinal function recovery after colorectal surgery: A meta-analysis. Zhonghua Wei Chang Wai Ke Za Zhi. 2013;16:1078-83.

17. Beyer-Berjot L, Patel V, Ziprin P, et al. Enhanced recovery simulation in colorectal surgery: Design of virtual online patients. Surg Endosc. 2015;29:2270-7.

18. Creamer F, Balfour A, Nimmo S, et al. Randomized open-label phase II study comparing oxycodone-naloxone with oxycodone in early return of gastrointestinal function after laparoscopic colorectal surgery. Br J Surg. 2017;104:42-51.

19. Gómez-Izquierdo JC, Feldman LS, Carli F, Baldini G. Meta-analysis of the effect of goal-directed therapy on bowel function after abdominal surgery. Br J Surg. 2015;102:577-89.

20. Shi WZ, Miao YL, Yakoob MY, et al. Recovery of gastrointestinal function with thoracic epidural vs. Systemic analgesia following gastrointestinal surgery. Acta Anaesthesiol Scand. 2014;58: 923-32

21. Naqvi SE, Zaka-Ur-Rab A, Islam N, Ali E. A prospective study of altered inflammatory response and its clinical outcome following laparoscopic and open cholecystectomy. Iran J Med Sci. 2017;42:347-53.

22. Glowka TR, Steinebach A, Stein K, et al. The novel CGRP receptor antagonist BIBN4096BS alleviates a postoperative intestinal inflammation and prevents postoperative ileus. Neurogastroenterol Motil. 2015;27:1038-49.

23. Gomez-Pinilla PJ, Binda MM, Lissens A, et al. Absence of intestinal inflammation and postoperative ileus in a mouse modelof laparoscopic surgery. Neurogastroenterol Motil. 2014;26:1238-47.

24. Lubbers T, Luyer MD, de Haan Jj, et al. Lipid-rich enteral nutrition reduces postoperative ileus in rats via activation of cholecystokinin-receptors. Ann Surg. 2009;249:481-7.

25. Lunding JA, Nordström LM, Haukelid AO, et al. Vagal activation by sham feeding improves gastric motility in functionaldyspepsia. Neurogastroenterol Motil. 2008;20:618-24.

26. Viswanathan C, Bhosale P, Ganeshan DM, et al. Imaging of complications of oncological therapy in the gastrointestinaPsystem. Cancer Imaging. 2012;12:163-72 